\title{
A New Low-Cost Bionic Hand
}

\section{Mr. TJ Brown, Middle Tennessee State University}

TJ Brown earned his Bachelor of Science in 2015 at Middle Tennessee State University where he studied Electro-Mechanical Engineering Technology and focused on researching new designs for bionic technology.

\section{Dr. Chong Chen P.E., Middle Tennessee State University}

Dr. Chong Chen is a professor in the Department of Engineering Technology at Middle Tennessee State University. He received B.S. degree from Hebei Institute of Technology in China, M.S. degree from Tianjin University in China, and Ph.D. degree from University of Kentucky, all in Electrical Engineering. Dr. Chen teaches electric circuits, electronics, controls, and industrial electricity. His research areas include controls, power electronics, electric machines, and electromagnetic fields. Dr. Chen is a Professional Engineer registered in the State of Tennessee. 


\title{
A New Low Cost Bionic Hand
}

\begin{abstract}
Technology has come a long way in the past three decades. Electronic devices have become cheaper and cheaper every year because of new advancements that help create smaller, faster, and more reliable devices. The more recent advancements in the field of prosthetic limbs have enabled patients to control bionic limbs using electrical signals that are generated from muscle contractions through a process called electromyography (EMG). However, these bionic limbs have not been around long and the costs associated with them are still too high to make them accessible to those who really need them.
\end{abstract}

This student research project utilizes recently acquired skills in Electro-Mechanical Engineering Technology to lower the price of a bionic hand. The mechanical parts that make up the hand are designed using 3D CAD software and then created on a 3D-printer. Using 3D-printing, the hand can easily be scaled to any size much more cheaply than using traditional methods. The project also lowers cost by designing and creating its own EMG circuit. This is the most important part of the whole system because it gives the user control over the hand. Without it, the hand is just for looks. The EMG circuit measures voltage across an arm muscle. When the muscle contracts, the circuit sends signals into a microcontroller that operates the 3D-printed hand with the help of a few servos.

There are some companies currently working on cheaper models of bionic hands as well, but most of these have limited functionality and the cost to buy them is still too high. The total cost of this bionic hand project up to this point is approximately $\$ 500$, compared to other models on the market today that sell for as much as a new car. Granted, this hand is not ready to replace a prosthetic hand just yet, but the built prototype was a complete success. The next step of the project is to focus on design improvements and to add another EMC circuit in order to read more muscles. This will allow the bionic hand to bring some real competition to the market and drive the price down.

\section{Introduction}

Prosthetic hands, or more appropriately bionic hands, have seen a lot of new development in the recent years. They no longer have to serve merely as a deterrent to keep people from staring. Nowadays, they can actually perform tasks such as holding objects, help tie shoes, and even type on keyboards. This is made possible because bionic hands are controlled using the electricity measured from muscle contractions through a process called electromyography (EMG). One of the top commercial brands on the market is Bebionic, their hands offer a much better quality of life to whoever can afford them with prices above $\$ 30,000^{[1]}$, but even this high dollar hand requires the user to select which grip function they want to preform before using the hand. They select functions by either pushing a button or manually repositioning the thumb. Having the user stop in the middle of a task in order to setup their hand for the next chore is not what most people would expect from a hand that cost as much as a car. 
There are other organizations out there who are trying to help bring the cost of bionic hands down by using a fairly new technology known as 3D-printing. These organizations are designing 3D-printable hands that users can download for free to use. These are referred to as open source projects and with 3D-printers becoming more affordable, prices starting around $\$ 1,000^{[2]}$, they could provide a huge advantage to parents that have to contend with a child who is constantly out growing a fitted hand.

However, most of these open source projects still require a lot more work than the typical user is capable of doing, because they still require electronics and programing before functioning as a bionic hand. Fully functional 3D-printed hands such as the Dextrus model start around $\$ 1,100$ [3]. Figure 1a is a picture of the lower cost Dextrus compared to the high dollar Bebionic brand in Figure 1b.

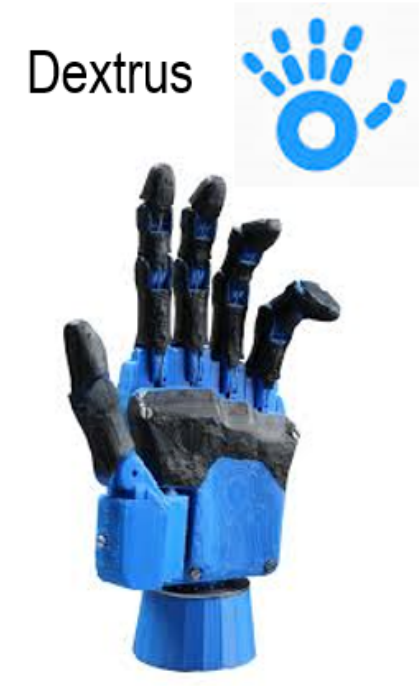

Figure 1a: A model of low-end bionic hands

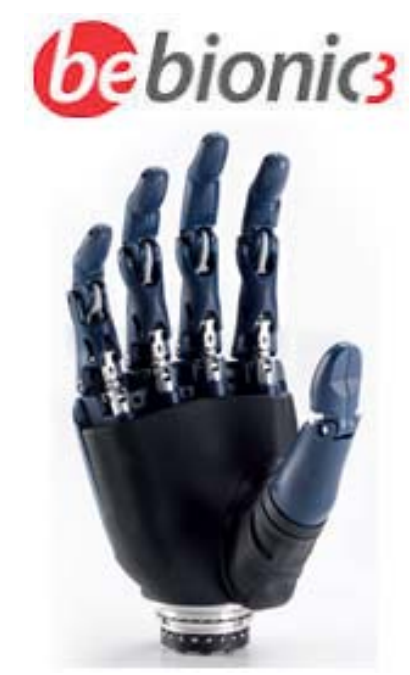

Figure 1b: A model of high-end bionic hands 
This project's purpose was to design, simulate, and create a new bionic hand from the ground up for under $\$ 1,000$ that is capable of generating some competition to drive the high dollar bionic hand brands down, so that people who really need them can afford them. This means designing, simulating, and building an EMG circuit. Then designing, simulating, and 3D-printing a new hand. Finally, connect the two parts together with a microcontroller that requires programming.

\section{Design and Fabrication}

The bionic hand electronics circuit consists of three main parts. It has a microcontroller for controlling, an EMG circuit for sensing, and servos for operating a 3D-printed hand. The microcontroller that was chosen for this design is the Basic Stamp 2 Homework board from Parallax. It is capable of receiving a signal from the EMG circuit and then outputting a signal to control three servos to operate the hand. The programing for the microcontroller is fairly simple in that it only has one input and one output to control.

The first EMG circuit was designed based on a book written by Muhammad Jamal called Signal Acquisition Using Surface EMG and Circuit Design Considerations for Robotic Prosthesis ${ }^{[4]}$. It provided important information that was used in designing the circuit for this project, such as the EMG circuit must be able to measure and amplify 1-10 millivolts at a frequency range between $0-500 \mathrm{~Hz}$. The first EMG circuit designed for this project is provided in Figure 2.

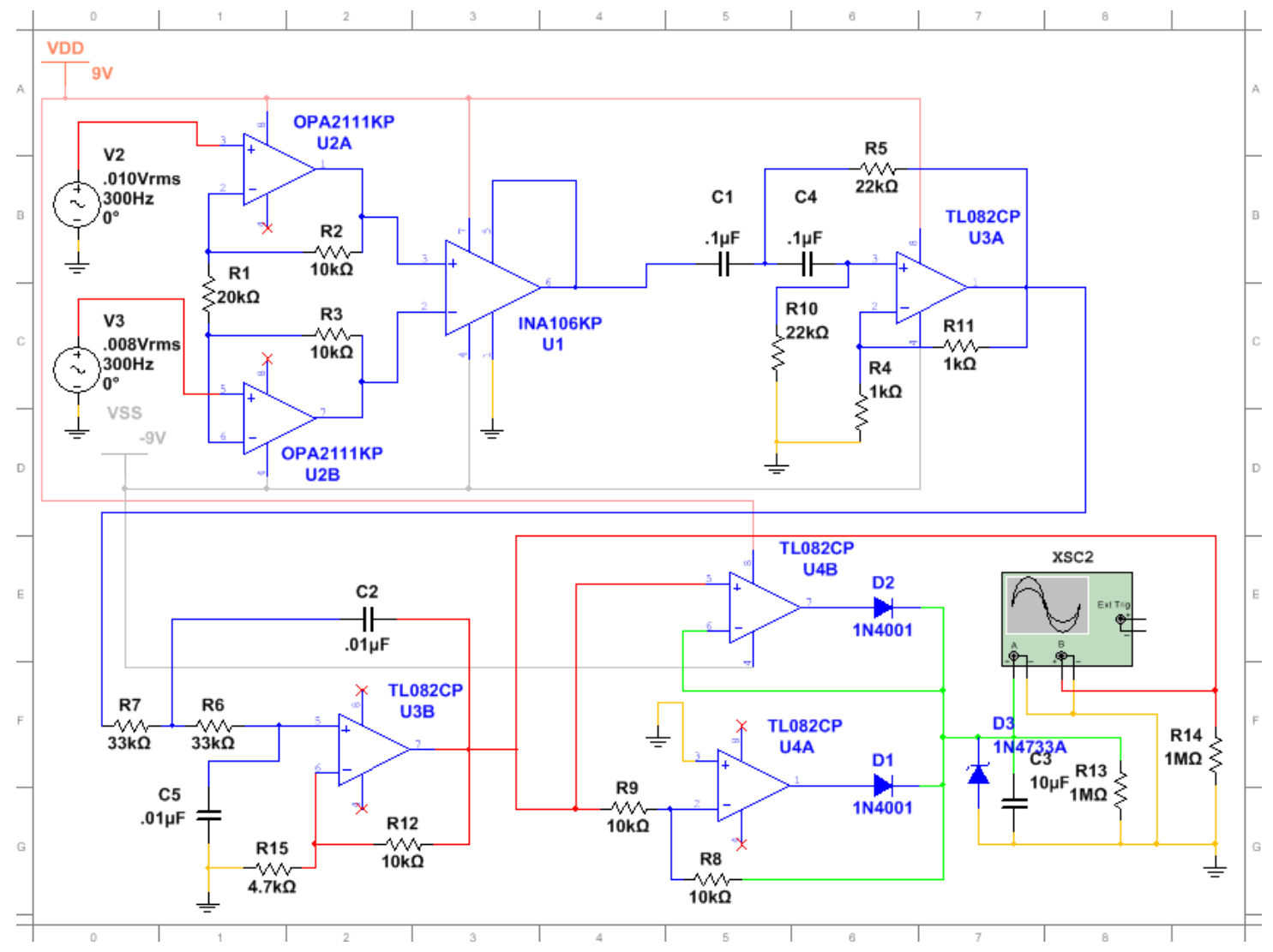

Figure 2: First EMG circuit 
This circuit is designed to amplify the voltage measured from two points located near the center of the bicep. Then the difference of these amplified voltages are filtered and rectified to produce a DC signal of approximately 3-5 volts. In Figure 2, the simulation uses V2 and V3 to represent the two points on a bicep. The simulation on the oscilloscope (XSC2 in Figure 2) can be seen in Figure 3. The oscillating waveform in Figure 3 is the signal after it has been amplified and filtered by the second order high pass and low pass filters. These filters allow a signal approximately between $70 \mathrm{~Hz}$ and $480 \mathrm{~Hz}$ to pass through the circuit and appear on the oscilloscope as the red signal. The output signal (green straight line) in Figure 3 is created after the red oscillating signal passes through the rectifier circuit. This is done to produce a DC signal from the oscillating signal so that it can be used as high and low voltages. The high voltage is used to confirm a muscle contraction at the microcontroller.

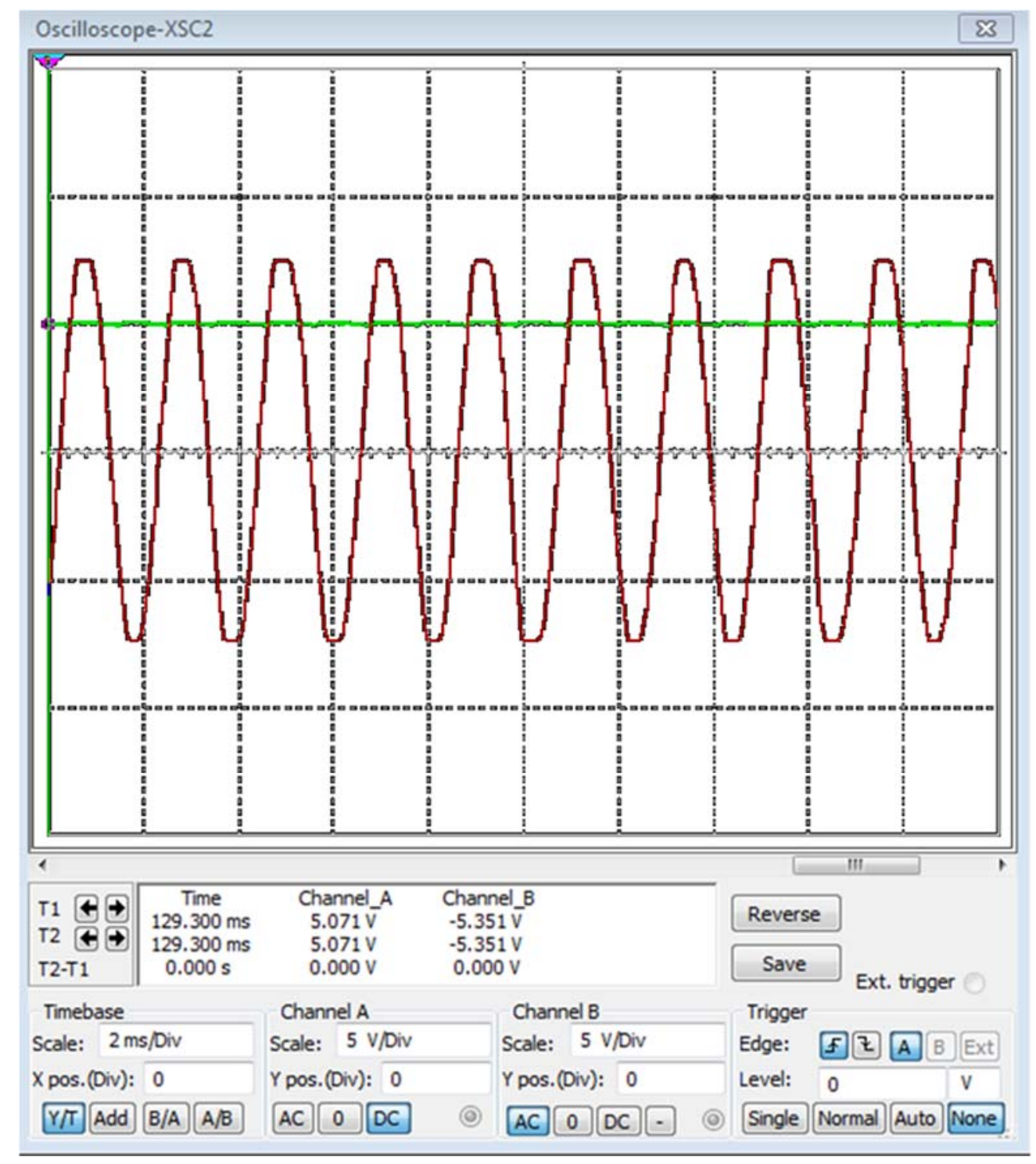

Figure 3: Simulation of first EMG circuit 
After testing the circuit, another design for an EMG circuit was also created and tested. The main differences in the first and second EMG circuits are: when the signals get amplified and how they are filtered. The first circuit amplifies and then takes the difference of the two points measured on the bicep, whereas the second circuit takes the difference of the two points before amplifying [4], [5], [6]. The second circuit was a better design because passing the signals first through the differential amplifier helps eliminate amplifying electrical noises that were not taken into consideration during the simulation phase. The second circuit also uses less components to filter the signal before rectifying it. High and low filters in the first circuit require twice the amount of capacitors and resistors compared to the second circuit. The second EMG design was chosen for this project because it proved to be more stable and simpler to produce. The schematic of this circuit can be seen in Figure 4 along with an additional power circuit.

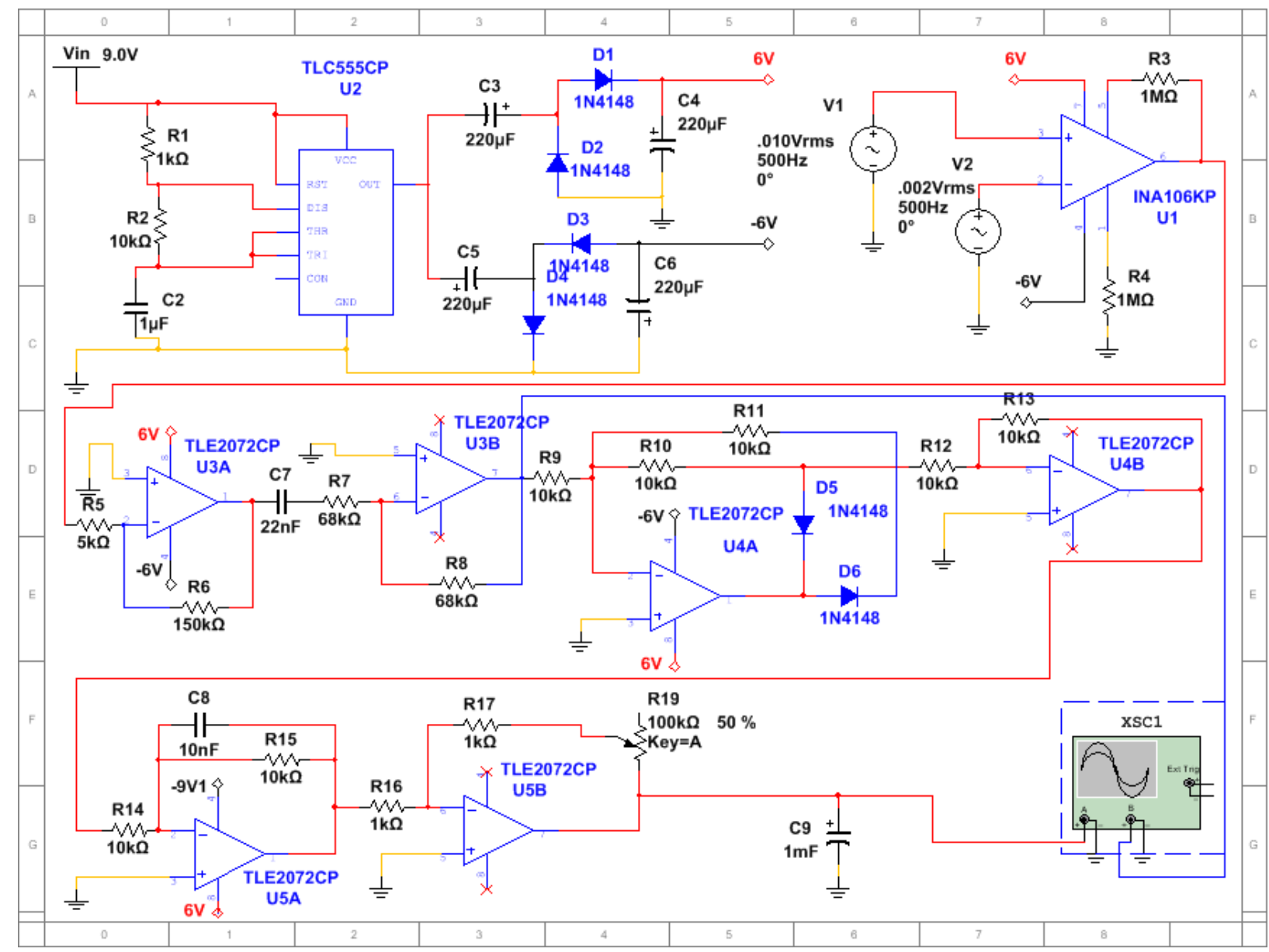

Figure 4: Final EMG circuit

This circuit uses a power supply designed from a NE555 timer chip represented in the schematic by the TLC555 [7], [8]. This allows the EMG circuit to be powered by one 9 volt battery that produces positive and negative voltages for the op-amps. The simulation of this EMG circuit on the oscilloscope (XSC1 in Figure 4) can be seen in Figure 5. The blue oscillating signal in Figure 5 is not as clean as the signal from Figure 3 because of using less components for the 
filters. Another side effect from building low grade filters is that the output signal (red line) is not as straight as the previous circuit. This signal has a slight wave to it, however it still works well enough to provide the high and low DC voltages needed to confirm muscle contractions so it was chosen as the final design for the EMG circuit. The picture of the built circuit can be seen in Figure 6 and all the values for the components are provided in Figure 4.



Figure 5: Simulation of Final EMG circuit 


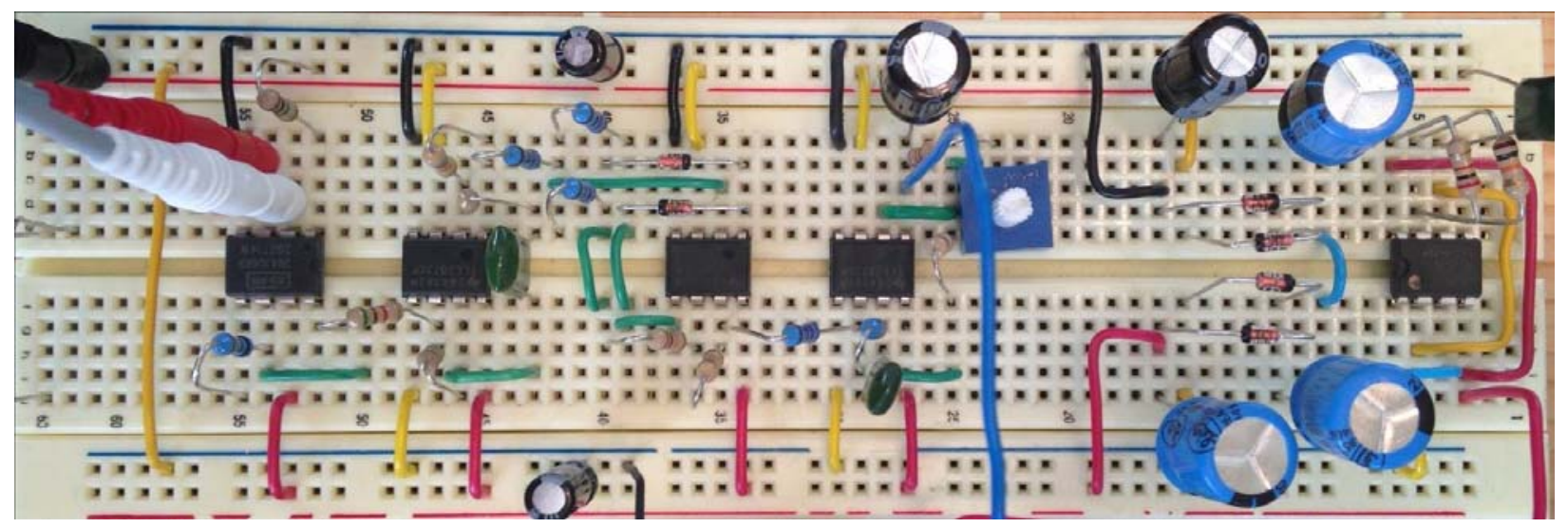

Figure 6: EMG circuit with power supply circuit

With a working EMG circuit designed, simulated, and created, the next part of the project focuses on the design of the hand. The idea was to use the same principles as a human hand but instead of using tendons high strength braided fishing line was used for its ability to hold a lot of tension. To keep the braided line from breaking under high stress, bands that are commonly used for braces in the orthodontics industry were designed into the system. Figure 7 provides the basis of how the hand was designed to work ${ }^{[9]}$. Notice in Figure 7 how the tendon represented by the arrow moves to the left and how it causes the fingertip to move. This project uses that same concept but neglects the joint at the tip of the finger because it is not necessary for most tasks.

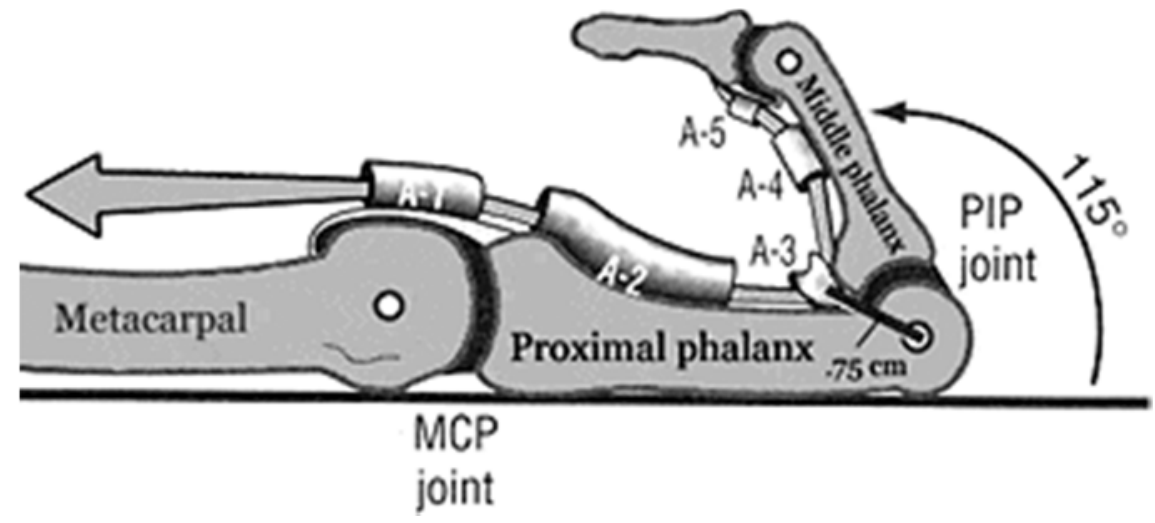

Figure 7: Basis for bionic finger ${ }^{[9]}$ Permission provided by Donald A. Neumann

A prototype finger was created on a 3D-printer out of acrylonitrile butadiene styrene (ABS plastic) to get an idea of tolerances needed for the joints before designing the entire hand. The prototype is shown in Figure 8. 


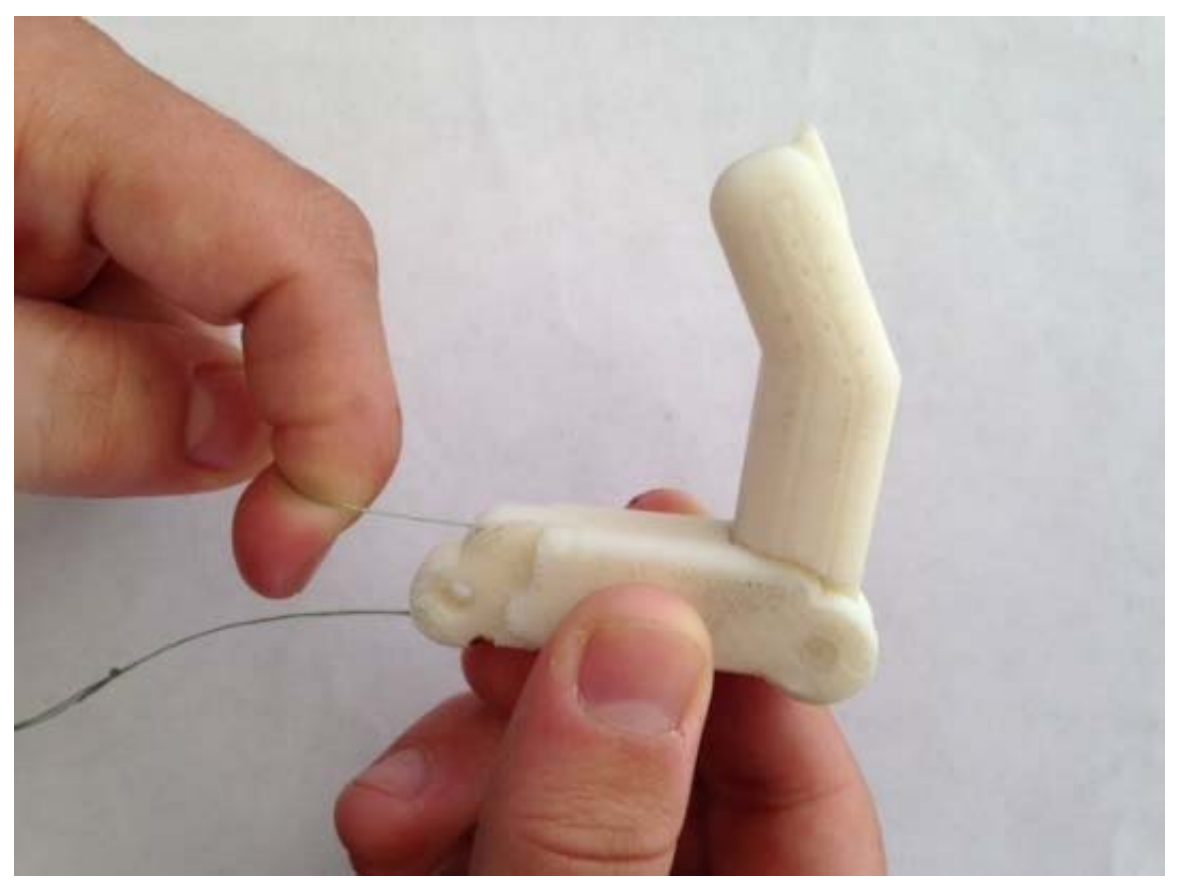

Figure 8: Prototype finger

After confirming that the joint designed for the prototype worked, a new finger design was created that would be easily scaled to other sizes for the other fingers. Once all the fingers were designed using Inventor 3D CAD software, the rest of the hand was created and all the components were assembled in an assemble file. This allowed the hand to be simulated before printing all the parts on the 3D-printer. An image of the complete hand being simulated can be seen in Figure 9. The hand was then printed on a 3D-printer and all of the components were strung up and pinned together.

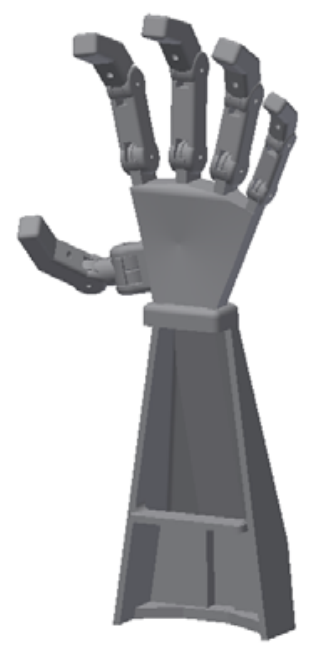

Figure 9: Simulation of printed parts for hand 
The servos used in this design are capable of producing seven pounds of tension for operating the fingers. The thumb and index finger were designed to have individual servos, while the last three fingers are controlled by one servo. Three servos are used so that when extra EMG circuits are added in the future the hand will be capable of more tasks. However, for this project one servo could have been used because it is only going to use one EMG circuit. Having only one input means this hand will only be able to perform two task: opening and closing the hand. Pictures of the assembled hand in the closed and opened form can be seen in Figure 10a and 10b.

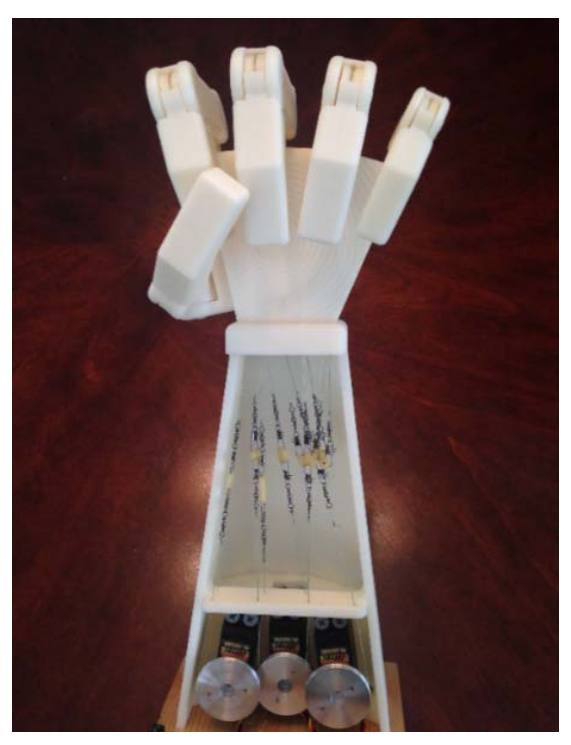

Figure 10a: Closed bionic hand

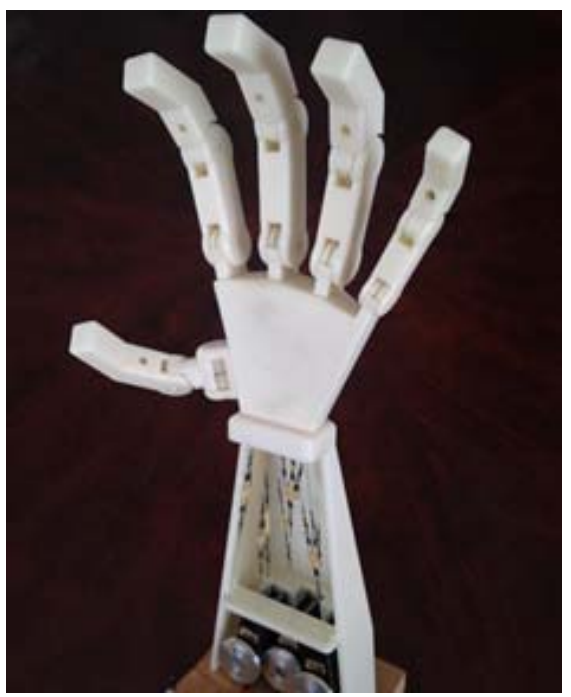

Figure 10b: Opened bionic hand 
With the hand completed, the next step was to connect the EMG circuit and the servos to the microcontroller. Then the microcontroller was programed to control the servos with the signal from the EMG circuit.

\section{Calibration and Test}

Once the microcontroller was programed, the bionic hand was then calibrated so that all the components functioned properly together as intended. Figure 11 shows the circuit being adjusted to output the signal from the EMG circuit above three volts when the demonstrator's hand is closed and a voltage below one volt when the demonstrator's hand is open.

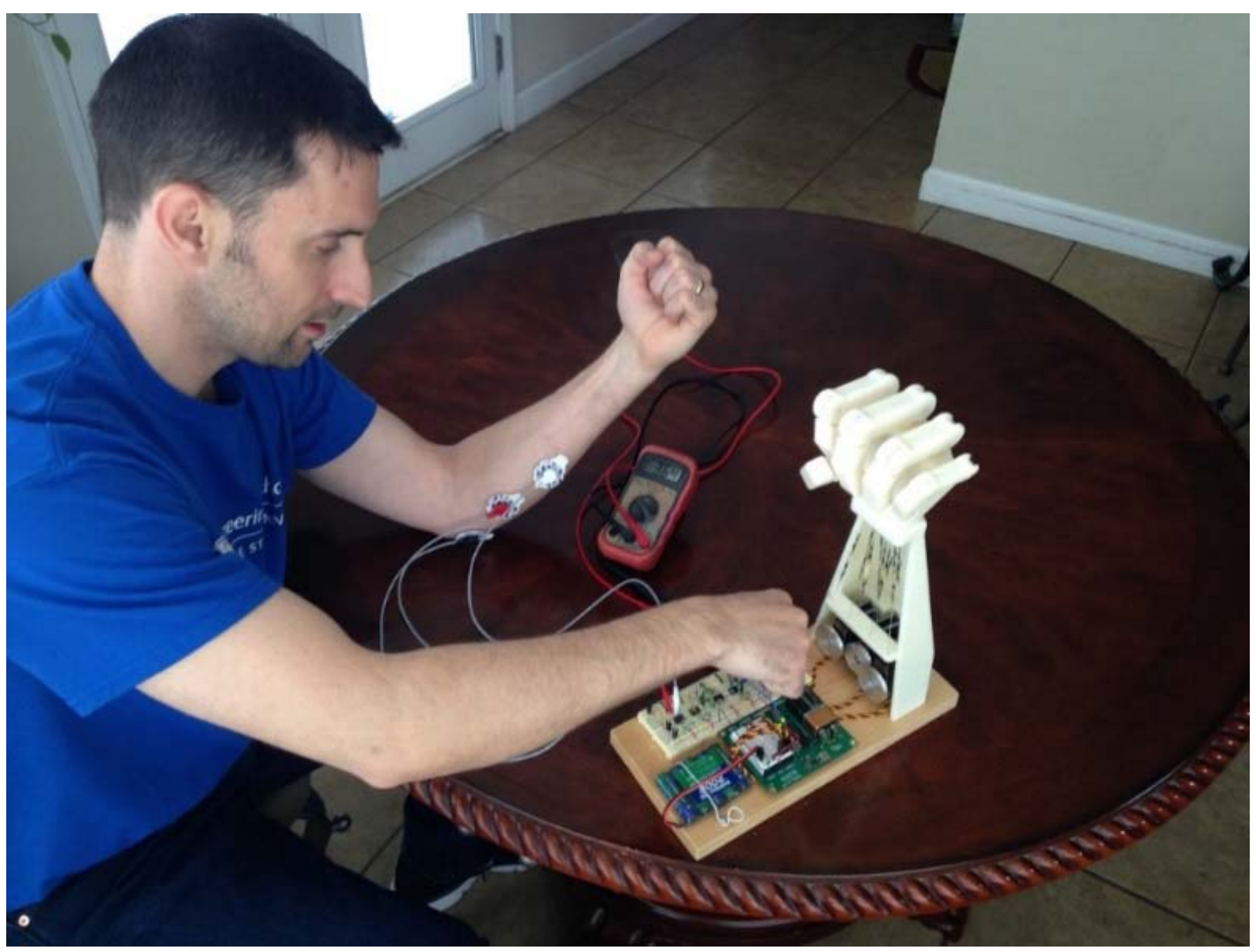

Figure 11: Calibration of the bionic hand

Then the microcontroller distinguishes these two voltages as high and low, which is represented as 1 and 0 . When the signal is high, the microcontroller pulses out a signal to the servos that causes them to turn 180 degrees. The pulleys on the servos then pull the cables of the fingers and thumb causing them to move to the closed hand position shown in Figure 10a.

\section{Conclusion}


The project was a complete success! Once the system was calibrated, the EMG circuit was verified to work on both the bicep and the forearm. The bionic hand has a reaction time less than 0.25 seconds. The speed of opening and closing the hand depends on how hard the muscle is flexed because the voltage amplified from the muscle has magnitude.

Through the use of Multisim simulation software, the design for the EMG circuit was flawless in providing a working schematic to build a prototype. Likewise, the Inventor 3D CAD software allowed the hand design to be built and tested to ensure all components fit properly together before printing them on the 3D-printer. Using a wide range of engineering technology, a working bionic hands was designed, created, and tested for a total cost of $\$ 525$. This is well under the $\$ 1,000$ goal. Granted, this hand is not ready to replace a prosthetic hand at this time, but now with a working prototype attention can be focused on improving it.

The size of the components were not considered when designing the hand, however, the EMG circuit and microcontroller can be commercially built together much smaller to make it more compactable. The amount of batteries used were also of no concern because these can also be reduced.

The next step for the project is to design the EMG circuit to operate with only a positive voltage supply for the op-amps and have the circuit commercially produced. This would reduce the size of both the circuit board and the battery space needed for the Bionic hand. The 3D-printed hand design should work just fine for the next step of the process. Another EMG circuit will be added so that two muscles can be monitored. The addition of the extra input will allow the hand to perform three more task, such as: individual index control, individual thumb control, and both index with thumb control for pinching objects.

\section{Bibliography}

[1] Mat Smith, New beBionic hand almost doubles its grip-strength, steered by user's electrical 'skin signals', www.engadget.com

[2] Top Ten Reviews, 3D Printers, 3d-printers.toptenreviews.com

[3] Darrel Etherington, The Dextrus Robotic Hand Wants To Make Advanced Prosthetics 100X Cheaper Via 3D Printing, techcrunch.com

[4] Jamal, Muhammad Zahak. Signal Acquisition Using Surface EMG and Circuit Design Considerations for Robotic Prosthesis. N.p.: INTECH Open Access, 2012. Print.

[5] Wang, Jingpeng, Liqiong Tang, and John E. Bronlund. "Surface EMG Signal Amplification and Filtering." International Journal of Computer Applications IJCA 82.1 (2013): 15-22. Web.

[6] C.J. De Luca. "Electromyography." Encyclopedia of Medical Devices and Instrumentation. John G. Webster ed. N.p.: John Wiley, 2006. 98-109. Print 
[7] "555 DC Boost Converter Circuits." Electronic Projects Circuits. N.p., 19 Aug. 2012. Web. 20 Mar. 2016

[8] Raja, Dilip. "Negative Voltage Generator Circuit Diagram Using IC 555." Circuit Digest. N.p., n.d. Web. 20 Mar 2016.

[9] Donald A. Neumann, Kinesiology of the Musculoskeletal System: Foundations for Rehabilitation, 2nd Edition, Mosby, December, 2010. 